IOS Press

\title{
Pediatric Rehabilitation Medicine certification
}

On November 15, 2016, the American Board of Physical Medicine and Rehabilitation (ABPMR) administered the examination for the subspecialty of pediatric rehabilitation medicine. Effective December 1, 2016, the following individuals were certified:

Akamagwuna, Unoma, Pittsburgh, PA;

Bartlett, Adam, Alameda, CA;

Camilleri, Jessica L, Fishers, IN;

Cho, Kelly Lynn, Minneapolis, MN;

Holman, Lainie, Cincinnati, $\mathrm{OH}$;
Kapadia, Mitul Ranjan, San Francisco, CA; Landry, Bradford William, Rochester, MN;

Luce, John, Berkeley, CA;

Murdock, Erin, Cleveland, OH;

Parker, Francisco, Cincinnati, OH;

Pham, Kelly Loraine Dauer, Seattle, WA;

Rojas, Ana-marie, Chicago, IL;

Sinn, Clarice Nicole, Houston, TX;

Sofair, Audrey Fawn, Armonk, NY;

Talley, Reginald Donte, Columbus, $\mathrm{OH}$. 